\title{
Germanica
}

\section{Le Nouveau Roman, pierre de touche de la modernité littéraire}

Der Nouveau Roman, ein Prüfstein der literarischen Moderne

The Nouveau Roman, a touchstone for literary modernity

\section{Solange Arber}

\section{OpenEdition}

\section{Journals}

Édition électronique

URL : http://journals.openedition.org/germanica/3339

DOI : 10.4000/germanica.3339

ISSN : 2107-0784

\section{Éditeur}

Université de Lille

Édition imprimée

Date de publication : 30 décembre 2016

Pagination : 19-32

ISBN : 9782913857384

ISSN : 0984-2632

\section{Référence électronique}

Solange Arber, « Le Nouveau Roman, pierre de touche de la modernité littéraire », Germanica [En ligne], 59 | 2016, mis en ligne le 30 décembre 2018, consulté le 06 janvier 2021. URL : http:// journals.openedition.org/germanica/3339; DOI : https://doi.org/10.4000/germanica.3339 


\title{
Le Nouveau Roman, pierre de touche de la modernité littéraire
}

\author{
Solange ARBER \\ Université Paris-Sorbonne, Université de Lausanne
}

En 1950, Ernst Robert Curtius écrivait : « seule la France nous enseigne ce qu'est la littérature ${ }^{1}$ ». L'idée de la France comme figure de proue de la littérature mondiale est encore vivante dans les années 1960. C'est d'ailleurs en citant Curtius que deux romanistes de part et d'autre du rideau de fer, Gerd Krause ${ }^{2}$ et Werner Krauss ${ }^{3}$, abordent la question la plus brûlante de l'actualité littéraire des dix dernières années : le Nouveau Roman. Ce dernier a connu une grande fortune critique, aussi bien internationale que française. Cela peut s'expliquer par plusieurs facteurs. L'idée d'une «école » du refus ou du regard et les interventions polémiques de son «pape » Alain Robbe-Grillet séduisent les médias et assurent la visibilité de ses membres. Plus profondément, la rupture radicale avec les normes du roman réaliste, le refus de notions constitutives du genre, comme le héros ou l'histoire, et surtout l'affirmation de l'autonomie de l'art, contribuent à faire du Nouveau Roman, ainsi

1. - Ernst Robert Curtius, Kritische Essays zur europäischen Literatur, Bern, A. Francke, 1950, p. 7. Toutes les traductions de citations sont de moi.

2. - Gerd Krause, Tendenzen im französischen Romanschaffen des zwanzigsten Jahrhunderts : nouveau Roman, traditioneller Roman, Frankfurt a.M., Moritz Diesterweg, 1962, p. 77.

3. - Werner Krauss, « Revolution des Romans? Bemerkungen zum "Nouveau Roman" », in Essays zur französischen Literatur, Berlin, Aufbau Verlag, 1968, p. 107. 
dénommé par Émile Henriot, le dernier avatar d'une modernité devenue le discours dominant de l'histoire littéraire, le « récit orthodoxe ${ }^{4}$ » de la critique.

Le Nouveau Roman a ainsi été considéré comme la forme la plus poussée du roman moderne depuis les années 1950 jusque dans les années 1970. Les prises de position qu'il inspire s'inscrivent donc dans le cadre du débat sur la modernité, à une période charnière où s'annonce la transition entre la modernité « classique »(klassische Moderne) du début du $\mathrm{xx}^{\mathrm{e}}$ siècle et la postmodernité. La question de la réception du Nouveau Roman par la critique dans l'espace germanophone devrait donc permettre de dévoiler certaines tendances du discours sur la modernité littéraire entre Allemagne de l'Ouest et de l'Est. En montrant les interactions entre trois champs littéraires, on espère remettre en question la vision dichotomique de l'opposition Est-Ouest. Pour ce faire, il s'agira de retracer le transfert du Nouveau Roman dans l'espace germanophone divisé, puis de comparer les positions des différents critiques qui ont participé à sa réception. Cette étude se fonde sur les publications d'ouvrages et d'articles de revue en allemand qui traitent du Nouveau Roman en général ou des quatre auteurs phares du mouvement - Alain Robbe-Grillet, Nathalie Sarraute, Claude Simon et Michel Butor - entre les années 1950 et 1990.

\section{La route des Allemagnes}

\section{Allemagne de l'Ouest}

Contrairement aux armées de 1940 dont Claude Simon raconte la débâcle, la route de l'Allemagne de l'Ouest ne passe pas par les Flandres, mais par la Suisse. C'est en effet la romaniste suisse Gerda Zeltner qui a joué un rôle prépondérant dans le transfert du Nouveau Roman, notamment avec son livre de 1960, Das Wagnis des französischen Gegenwartsromans, qui fait figurer Sarraute, Robbe-Grillet et Butor en bonne place au milieu de Camus, Malraux et Sartre. Véritable passionnée, elle a multiplié les publications sur le sujet, qu'il s'agisse de livres, de conférences ou d'articles du Feuilleton à l'occasion de nouvelles parutions en français ou en allemand 5 . Les titres choisis reflètent le lien étroit qui est fait entre Nouveau Roman et modernité littéraire : Im Augenblick der Gegenwart : moderne Formen des französischen Romans (1974), Was ist ein moderner Roman? : Versuch einer Standort-bestimmung (1972).

4. - Antoine Compagnon, Les cinq paradoxes de la modernité, Paris, Éd. du Seuil, 1990, p. 56 sq.

5. - Cf. Gerda Zeltner-Neukomm, Die eigenmächtige Sprache: zur Poetik des Nouveau Roman, Olten, Walter-Verlag, 1965. 
Gerda Zeltner ne s'adresse pas qu'à un public de romanistes : ses livres ne sont pas publiés dans des collections spécialisées et les citations sont traduites en allemand. C'est pour cette raison que son travail a rencontré un grand écho et a énormément contribué à asseoir le Nouveau Roman dans l'horizon littéraire ouest-allemand. La revue Akzente de Munich, dirigée par Walter Höllerer, a également joué un rôle déterminant dans ce transfert à partir de 1956. En 1958, elle a consacré un numéro aux théories de Robbe-Grillet et de Sarraute, puis un deuxième, réclamé par les lecteurs : «Suite à de nombreuses demandes, Akzente poursuit le débat lancé dans le premier cahier de cette année sur les possibilités du roman moderne ${ }^{6} \gg$. Dès le début, l'intérêt pour le Nouveau Roman s'inscrit dans le cadre d'un débat général sur la modernité et dépasse donc le cercle des romanistes. Ceux-ci apparaissent même légèrement en retard, puisque ce n'est qu'à partir de 1962 que Kurt Wilhelm commence à publier des articles présentant les différents protagonistes du mouvement dans la Zeitschrift für französische Sprache und Literatur ${ }^{7}$. Les innovations d'outre-Rhin attisent la curiosité du monde littéraire de RFA. C'est à la demande de la maison d'édition Moritz Diesterweg que le romaniste Gerd Krause se charge, à l'évidence contre ses goûts personnels, de présenter au public ouest-allemand le roman français moderne ${ }^{8}$. Cette curiosité est alimentée par le Feuilleton, dont les articles touchent un large public. Les déclarations péremptoires d'un Marcel Reich-Ranicki contre le Nouveau Roman ont autant contribué à faire connaître ce dernier que toutes les publications mentionnées précédemment. Dans le camp adverse, ce sont Reinhard Baumgart et Helmut Scheffel qui ont assumé la lourde tâche de défendre les auteurs frappés par un anathème aussi puissant.

Malgré les polémiques, on peut parler dans le cas de la RFA d'un transfert réussi, dans la mesure où le Nouveau Roman a fait l'objet, à partir des années 1960 et jusque dans les années 1990, de publications nombreuses touchant un public au-delà du cercle restreint de la romanistique. On peut dénombrer 18 ouvrages de littérature secondaire publiés dans l'espace germanophone de l'Ouest entre les années 1960 et

6. - Walter Höllerer et Hans Bender (éd.), « Der Held des Romans und die Erzählform », Akzente, 1958, p. 24.

7. - Kurt Wilhelm, "Der "Nouveau Romancier" Alain Robbe-Grillet », Zeitschrift für französische Sprache und Literatur, 72-3/4, 1er janvier 1962, p. 169-185 ; Kurt Wilhelm, "Nathalie Sarrautes "Nouveau Roman" », Zeitschrift für französische Sprache und Literatur, 74-4, 1er janvier 1964, p. 289-320 ; Kurt Wilhelm, "Claude Simon als "Nouveau Romancier" », Zeitschrift für französische Sprache und Literatur, 75-4, 1965, p. 309-352 ; Kurt Wilhelm, « Michel Butors Romankonzeption », Zeitschrift für französische Sprache und Literatur, 74-1, 1964, p. 1-21.

8. - G. Krause, Tendenzen im französischen Romanschaffen des zwanzigsten Jahrhunderts..., op. cit. 
$1990^{9}$. C'est dans la première décennie que le nombre de publications est le plus important, avec une majorité de livres s'adressant à un public de non spécialistes. Dans les années 1970, le nombre de publications baisse un peu et compte une part plus importante de livres s'adressant à des romanistes ou des germanistes. L'existence d'ouvrages abordant le Nouveau Roman dans des collections de germanistique peut étonner ; elle témoigne de l'adoption de ce dernier comme mètre étalon de la modernité littéraire par certains chercheurs en littérature allemande. Avec Zwei deutsche Literaturen? d'Eberhard Mannack (1977), le Nouveau Roman est érigé en arbitre de la question de l'unité de la littérature allemande dans une Allemagne divisée. Mais c'est surtout dans les années 1990 que l'on trouve un nombre relativement important d'ouvrages de germanistes faisant référence au Nouveau Roman. En 1991, Jürgen Petersen lui consacre tout un chapitre dans Der deutsche Roman der Moderne ${ }^{10}$. Ses efforts pour rattacher des écrivains germanophones contemporains au Nouveau Roman témoignent de la volonté d'intégrer ce mouvement important du roman moderne dans l'histoire littéraire allemande.

\section{Allemagne de l'Est}

Cette volonté de se rattacher au Nouveau Roman est complètement absente en RDA. Dans le cadre d'un monde littéraire soumis à la politique culturelle du régime communiste, il n'est guère possible de se revendiquer d'un mouvement qui rejette la conception du réalisme sur laquelle se fonde l'esthétique officielle, et qui tombe manifestement sous le coup du formalisme. Le transfert, dans le cas de la RDA, parait devoir moins relever de choix individuels et de la demande du public que de décisions en haut lieu et du climat général de « gel » ou de « dégel ». Pourtant, cette idée est à nuancer. Si l'on regarde le détail des événements, il n'est pas toujours possible de trouver une corrélation entre les directives politiques et la réception du Nouveau Roman. En effet, la première publication est-allemande de romans de Michel Butor et de Nathalie Sarraute date de 1966. Or c'est peu de temps après la onzième réunion plénière du comité central du SED de 1965 qui marque un durcissement de la politique culturelle ${ }^{11}$. Cela s'explique par le fait que la vie littéraire de la RDA est aussi influencée par le contexte plus général du Bloc de l'Est : les années précédentes, deux conférences internatio-

9. - Ces données rassemblent les publications les plus importantes, sans prétendre à l'exhaustivité.

10. - Jürgen H. Petersen, Der deutsche Roman der Moderne: Grundlegung, Typologie, Entwicklung, Stuttgart, J.B. Metzlersche Verlagsbuchhandlung, 1991.

11. - Cf. Rolf Grimminger et Hans-Jürgen Schmitt (dir.), Die Literatur der DDR, München, C. Hanser Verlag, 1983. 
nales organisées dans des pays communistes ont en effet invité Michel Butor, Alain Robbe-Grillet et Nathalie Sarraute pour qu'ils s'expriment sur la question de la modernité. Ces manifestations se sont tenues à l'initiative d'associations internationales d'écrivains, mais n'auraient pas été possibles sans un soutien politique. En 1963, la COMES a organisé à Leningrad un colloque sur le roman moderne avec l'aide de l'Union des écrivains soviétiques, puis en 1964 une table ronde du PEN Club international a eu lieu à Budapest sur le thème du rapport entre tradition et modernité12. Dans son article «L'année dernière à Leningrad », Bernard Pingaud résume le colloque de 1963 par la formule du «paradoxe du dialogue recherché quoiqu'impossible ${ }^{13} »$. En effet, certains intervenants soviétiques ont adressé une fin de non-recevoir aux écrivains occidentaux, tandis que Robbe-Grillet a démontré une fois de plus son art de la provocation :

Le romancier est-allemand qui a déclaré, à cette tribune, faire des romans pour lutter contre le fascisme, me fait bien rire et me donnerait des inquiétudes sur ses qualités d'écrivain si nous ne comprenions pas, nous, que celui-là non plus ne sait pas pourquoi il écrit et que ses alibis n'ont aucune importance ${ }^{14}$.

Malgré l'impossibilité de concilier des conceptions de la littérature que tout oppose, le dialogue n'en est pas moins enclenché. Il devient acceptable de parler du Nouveau Roman, bien qu'il faille se garder de le présenter sous un jour trop favorable.

C'est par la revue Sinn und Form que le public de l'Allemagne de l'Est peut rencontrer le Nouveau Roman pour la première fois. Cette revue n'est pas soumise à un contrôle très strict de l'État en raison d'un tirage très faible, elle est d'ailleurs diffusée à l'international. Néanmoins, on n'y trouve aucune référence aux auteurs du Nouveau Roman avant 1965. Les écrivains français à l'honneur sont Aragon, Rolland et Éluard. Dans un article d'Ilya Ehrenburg, farouche thuriféraire soviétique du réalisme socialiste, on peut lire une attaque voilée contre la nouvelle scène littéraire française, qu'il ne prend toutefois pas la peine ou le risque de mentionner explicitement :

12. - Cf. Nicole Racine, «La COMES (1958-1969), une association d'écrivains dans la guerre froide », in : Jean-François Sirinelli et Georges-Henri Soutou (dir.), Culture et guerre froide, Paris, PUPS, 2008.

13. - Bernard Pingaud, «L'année dernière à Léningrad », Esprit, 329 (7), 1964, p. 16.

14. - Alain Robbe-Grillet, "L'écrivain, par définition, ne sait où il va, et il écrit pour chercher à comprendre pourquoi il écrit », Esprit, 329 (7), 1964, p. 64. Il ne m'a pas été possible de déterminer qui était l'écrivain est-allemand en question. 
Je sais que beaucoup d'auteurs ont arrêté de montrer des êtres humains pour remplacer le dévoilement des caractères humains par des conversations sur soi-même, par des discussions de concepts abstraits, par des bavardages de futilités mondaines ${ }^{15}$.

C'est donc seulement en 1965 que les choses changent, quand Sinn und Form publie certaines interventions de la table ronde de Budapest sur le thème « Tradition et modernité dans la littérature de notre temps », où figurent aussi bien Michel Butor qu'Alfred Kurella. En 1966 une série d'articles est consacrée au roman moderne, et notamment à Michel Butor à l'occasion de la publication de La Modification. Ces articles reconnaissent l'importance du Nouveau Roman pour la critique littéraire, tout en prenant leurs distances avec lui.

Si Sinn und Form reste prudemment dans le cadre de ce qui est acceptable en se contentant de présenter le phénomène sans le cautionner, l'éminent romaniste Werner Krauss prend plus de risques dans son article paru en 1968 « Revolution des Romans? Bemerkungen zum "Nouveau Roman"16 ». Il y défend le Nouveau Roman contre le reproche de formalisme en établissant un parallèle entre la déconstruction du personnage et de l'histoire et un monde occidental de plus en plus dépersonnalisé, reprenant l'idée de l'homme comme «matricule » avancée par Robbe-Grillet. Mais il ne s'arrête pas là : il ose suggérer que cette réalité du monde occidental moderne pourrait bien ne pas être absente du monde socialiste, justifiant donc le transfert du Nouveau Roman en Allemagne de l'Est. Il n'oublie cependant pas de couvrir ses arrières : "Nous sommes convaincus que toutes les littératures doivent prendre connaissance des expériences du "nouveau roman", sans pour autant que ces créations puissent prétendre à une application universelle ${ }^{17}$ ». La défense du Nouveau Roman par Werner Krauss est restée lettre morte, dans la mesure où les publications se sont taries dans les années 1970, pour ne reprendre que dans les années 1980 sous l'impulsion et presque du seul fait de Brigitte Burmeister.

Cette romaniste déploie une grande activité : elle publie Claude Simon, Alain Robbe-Grillet et Nathalie Sarraute, ainsi que la première monographie est-allemande sur le Nouveau Roman ; elle va même jusqu'à écrire un roman au style influencé par ce mouvement littéraire ${ }^{18}$. Elle ne se montre pourtant pas particulièrement tendre avec lui dans Streit um den Nouveau Roman (1983), où elle reprend les reproches

15. - Ilja Ehrenburg, « Französische Notizen », Sinn und Form, 1, 1961, p. 49.

16. - W. Krauss, « Revolution des Romans? Bemerkungen zum "Nouveau Roman" »..., op.cit., p. 127.

17. - Ibid.

18. - Brigitte Burmeister, Anders oder Vom Aufenthalt in der Fremde, Berlin, Verlag der Nation, 1987. 
de la critique marxiste : élitisme, recherche de l'autonomie esthétique, problème du réalisme, « refus de mettre les textes littéraires au service du dialogue social et de la mise en mouvement de la société19 ». Si le ton reste prudent, mettant l'accent sur la nécessité de combler une lacune de la recherche sans avancer de jugement quant à l'intérêt intrinsèque du phénomène pour le contexte est-allemand, l'auteure s'efforce néanmoins de libérer le Nouveau Roman de certaines accusations, notamment sur son rapport à la société capitaliste et sur la question du réalisme ${ }^{20}$. Le cas de Brigitte Burmeister montre bien les ambiguiités du transfert du Nouveau Roman en RDA : freiné et limité par la ligne idéologique officielle, il a toutefois été possible de publier sur le sujet, et même de s'en inspirer pour sa propre création littéraire, sous réserve de prêter allégeance à la critique marxiste et de respecter ses codes rhétoriques.

\section{La réception du Nouveau Roman}

\section{Les écrivains germanophones à l'aune du Nouveau Roman}

Les critiques et les historiens ouest-allemands ont eu tendance à comparer, voire à assimiler certains auteurs germanophones au Nouveau Roman. Cette tendance a été étudiée par Uwe Neumann dans son ouvrage Uwe Johnson und der Nouveau Roman (1992), où il revient sur le cliché faisant de «l'écrivain des deux Allemagnes » le « Nouveau Romancier allemand ». Neumann donne un panorama détaillé de la réception du Nouveau Roman dans le milieu du Feuilleton et de la critique littéraire, d'où il ressort que, le plus souvent, « [1]'étiquette Nouveau Roman est vidée de son contenu et finit par ne plus représenter qu'un synonyme de "narration non-traditionnelle" ou "difficile"21 ». En effet, si la question «Que pensez-vous du Nouveau Roman? » est une tarte à la crème des interviews des années 1960, la connaissance du phénomène est souvent superficielle ${ }^{22}$. Cela n'empêche pas écrivains et journalistes de s'exprimer sur le sujet, la plupart du temps avec sévérité et même avec méfiance ${ }^{23}$. Ainsi Alfred Andersch, Jean Améry et Marcel Reich-Ranicki sont-ils des adversaires farouches de cette nouvelle forme d'écriture moderne.

Dans ce contexte, les rapprochements entre les œuvres de certains écrivains germanophones et le Nouveau Roman apparaissent souvent un

19. - Brigitte Burmeister, Streit um den Nouveau Roman : eine andere Literatur und ihre Leser, Berlin, Akademie-Verlag, 1983, p. 9.

20. - Cf. ibid., p. 96 sq.

21. - Uwe Neumann, Uwe Johnson und der Nouveau Roman: komparatistische Untersuchungen zur Stellung von Uwe Johnsons Erzählwerk zur Theorie und Praxis des Nouveau roman, Francfort, P. Lang, 1992, p. 18.

22. - Ibid., p. 31-32.

23. - Ibid., p. 466 sq. 
peu légers, voire forcés. Dans Der deutsche Roman der Moderne, Jürgen Petersen consacre tout un chapitre au Nouveau Roman, sous prétexte qu'il s'agit de « ce type de roman moderne développé en France qui est le seul à avoir réussi à s'imposer dans la conscience littéraire de l'Europe comme genre poétique ${ }^{24} \gg$. Mais ses tentatives pour montrer comment le roman allemand s'inscrit dans un tel «genre » ne sont pas très convaincantes, car ses arguments finissent par se résumer à « l'amour du détail [...], que le Nouveau roman a introduit dans la littérature allemande de la modernité ${ }^{25}$ ». Il n'y a guère que Peter Handke à l'Ouest et Christa Wolf à l'Est qui aient brièvement revendiqué l'influence de Robbe-Grillet ou de Sarraute. La critique a néanmoins établi des parallèles ou détecté des similitudes avec les œuvres de Uwe Johnson, Max Frisch, Thomas Bernhard et Peter Weiss. Elle cite également Dieter Wellershoff et son école du « nouveau réalisme » de Cologne, Helmut Heißenbüttel et le cercle de Graz du côté de la poésie concrète, qui représentent une génération plus jeune et plus portée sur l'expérimentation formelle. Sigrid Schmid-Bortenschlager résume ainsi la situation :

Le Nouveau Roman et sa théorie fascinaient et inquiétaient les écrivains allemands dans les années 1960. [...] Les écrivains établis se sentaient personnellement visés par les attaques contre le roman traditionnel et se mettaient - comme il est compréhensible d'un point de vue psychologique - sur la défensive. Seule la jeune génération se montrait impressionnée et suivait le mouvement en partie aveuglément ${ }^{26}$.

Mais les raisons pour lesquelles les écrivains germanophones ont, dans leur grande majorité, fait preuve de beaucoup de méfiance envers le Nouveau Roman ne peuvent pas se réduire à une question psychologique. Uwe Neumann y voit plutôt la conséquence de la situation spécifique de la littérature allemande après 1945, pour laquelle l'art doit avoir une dimension éthique. Le refus des nouveaux romanciers d'entrer sur le terrain de la morale et de l'engagement politique est irrecevable dans un contexte de rupture avec les compromissions du passé nazi. Cela explique pourquoi les auteurs dont les similitudes avec le Nouveau Roman sont soulignées par la critique viennent majoritairement de Suisse ou d'Autriche : " Déchargée dans une large mesure du devoir de surmonter le passé nazi, la littérature de ces pays a offert un espace

24. - J.H. Petersen, Der deutsche Roman der Moderne..., op. cit., p. 333.

25. - Ibid., p. 346.

26. - Sigrid Schmid-Bortenschlager, Konstruktive Literatur : gesellschaftliche Relevanz und literarische Tradition experimenteller Prosa-Großformen im deutschen, englischen und französischen Sprachraum nach 1945, Bonn, Bouvier, 1985, p. 121. 
disponible pour les expérimentations littéraires bien avant que cela pût être le cas en Allemagne27 ».

L'obstination des critiques, puis des historiens de la littérature, à positionner certains auteurs par rapport à ce mouvement français qui n'a pourtant guère fait d'émule outre-Rhin, s'explique par le fait que, malgré toutes les réserves qu'ils pouvaient avoir à son sujet, ils lui reconnaissaient une valeur paradigmatique. Les prises de position sur le Nouveau Roman s'inscrivent dans le cadre du « récit orthodoxe de la tradition moderne » tel que le décrit Antoine Compagnon à partir de l'ouvrage célèbre de Hugo Friedrich ${ }^{28}$. Le concept de dépersonnalisation est toujours mis en avant et le plus souvent traité comme la conséquence de la crise du sujet à l'époque moderne ${ }^{29}$. Parce qu'il est inséré dans ce discours, le Nouveau Roman fonctionne comme pierre de touche de la modernité littéraire qu'il incarne.

\section{Points d'achoppement}

L'idée de dépersonnalisation a aussi été évoquée par le romaniste est-allemand Werner Krauss ${ }^{30}$ et témoigne des nombreux recoupements entre les discours de la critique à l'Est et à l'Ouest. En dépit des divergences face à la modernité littéraire et donc au Nouveau Roman, considéré comme son fer de lance, on constate en effet que ce sont toujours les mêmes points d'achoppement qui reviennent : le rapport à la réalité, le rapport au lecteur, le rapport à l'humanisme.

Quand la revue Akzente présente pour la première fois le Nouveau Roman au public germanophone de l'Ouest en 1956, c'est dans le cadre d'un dossier intitulé « Les écrivains face à la réalité ». Ce thème était en fait celui d'une rencontre entre écrivains français et allemands qui a eu lieu à Vézelay à l'initiative de la revue Documents un peu plus tôt cette même année, et dont les interventions sont publiées sous une forme abrégée par Akzente. La question du réalisme figure donc à l'initiale du transfert du Nouveau Roman dans l'espace germanophone. Il est important de noter à ce propos que l'initiative est venue du côté français : c'est une revue française qui a invité des auteurs germanophones à venir débattre d'une question qui ne se trouvait pas encore au centre des débats en Allemagne. Ainsi que l'observe Uwe Neumann, le débat sur le réalisme ne commence en Allemagne de l'Ouest véritablement

27. - U. Neumann, Uwe Johnson und der Nouveau roman..., op. cit. p. 505.

28. - A. Compagnon, Les cinq paradoxes de la modernité ..., op. cit., p. 60.

29. - Gerd Krause résume par exemple le Nouveau Roman à la notion d'« individualisme sceptique », cf. G. Krause, Tendenzen im französischen Romanschaffen des zwanzigsten Jahrhunderts..., op. cit. p. 8.

30. - Cf. supra. 
qu'à partir des années 1960 suite à la réception du Nouveau Roman ${ }^{31}$. C'est d'ailleurs ce qui ressort du dossier d'Akzente « Les écrivains face à la réalité », où les contributions des auteurs allemands soit n'abordent pas de front la question (par exemple Günter Eich qui préfère s'interroger sur la notion de réalité), soit lui adressent une fin de non-recevoir, tel Karl Korn qui affirme que les écrivains ouest-allemands rejettent la notion de réalisme qu'ils considèrent comme un « $\operatorname{slogan}^{32}$ » vide de sens. En filigrane, c'est bien sûr la relation à l'Allemagne de l'Est qui est en jeu, où le réalisme est considéré comme la seule forme d'art valable, par opposition à l'art « décadent » et « formaliste» de la modernité. Face à ce frère ennemi de la littérature allemande, les écrivains de l'Ouest, et notamment ceux du groupe 47 , ont été réticents à se confronter à la question du réalisme, que ce soit pour se distancier de la grande tradition romanesque ou pour la reprendre à leur compte. Le transfert du Nouveau Roman et des débats qu'il suscite en France va donc jouer le rôle de catalyseur en forçant la critique et certains écrivains de RFA à prendre position. Le dossier d'Akzente, pourtant très court, leur fournit les repères nécessaires, avec des expressions comme le « réalisme de la surface » de Roland Barthes ou le « réalisme de la présence » de RobbeGrillet, et bien sûr cette citation reprise à l'envi : " Le monde n'est ni signifiant, ni absurde. Il est, tout simplement ${ }^{33} »$.

Une fois le débat lancé en Allemagne de l'Ouest, il se déroule de manière similaire à ce qui se passe en France et à ce qui se passera à l'Est quelques années plus tard. Les mêmes arguments se font face, bien qu'ils soient utilisés dans des constellations différentes. Ainsi la critique conservatrice et la critique marxiste se rejoignent-elles pour souligner le rapport entre la modernité radicale de l'écriture du Nouveau Roman et le contexte historique et intellectuel de sa production. Pierre de Boisdeffre côté français et Gerd Krause côté allemand soulignent le scepticisme d'une littérature qui renonce à dire la réalité et l'expliquent par le pessimisme de la société française post-Libération, et plus généralement par l'individualisme privé d'assise du monde moderne. L'approche marxiste inaugurée en 1964 par Lucien Goldmann dans Pour une sociologie du roman, part aussi du principe que la littérature est le reflet des conditions socio-historiques de sa production, ce qui est une variation de la Widerspiegelungstheorie ${ }^{34}$. Cette analyse arrive à point nommé pour les

31. - U. Neumann, Uwe Johnson und der Nouveau roman..., op. cit., p. 489.

32. - Karl Korn, « Darstellung der Grunderfahrungen », Akzente, 3-4, 1956, p. $307-311$.

33. - Alain Robbe-Grillet, « Für einen Realismus des Hierseins », Akzente, 3-4, 1956, p. 315-3018.

34. - Michael Rother, Das Problem des Realismus in den Romanen von Alain Robbe-Grillet, Heidelberg, Winter, 1980, p. 91. 
romanistes est-allemands désireux de justifier la réception du Nouveau Roman en RDA. Werner Krauss s'en empare dans son essai de 1968 «Revolution des Romans » : les mots-clés de « dépersonnalisation » et de « réification » de la société occidentale dans sa «phase post-impérialiste de capitalisme de monopole à coopération mondiale ${ }^{35} \gg$ lui permettent d'argumenter en faveur d'une littérature qui, si elle ne répond pas au standard du réalisme prôné par la politique culturelle, n'en constitue pas moins une réponse à ces mutations profondes du monde moderne.

La réification de tous les rapports est un fruit du capitalisme tardif. En conséquence, Robbe-Grillet a totalement refondé le rapport entre homme et chose. [...] Si l'on fait le bilan de leur production on peut constater que le groupe informel des représentants du « nouveau roman » a parfois atteint une inhabituelle qualité de création littéraire. De nouveaux horizons ont été ouverts au roman, et à travers l'horizon du roman c'est de surcroît la connaissance de la réalité qui s'est approfondie ${ }^{36}$.

La réception du Nouveau Roman en Allemagne de l'Est comme de l'Ouest s'est focalisée sur la question du réalisme, comme le montrent notamment les thèses soutenues par Carola Gerlach (RDA) ${ }^{37}$ et Michael Rother (RFA) ${ }^{38}$ ainsi que les articles de Winfried Wehle (RFA) ${ }^{39}$ et de Brigitte Burmeister (RDA) ${ }^{40}$. Dans le cas de la RDA, cela s'explique par le fait que, selon Danielle Risterucci-Roudnicky, « le débat de fond de la littérature est explicitement et implicitement celui du réalisme ${ }^{41} »$. Mais c'est aussi et surtout pour des raisons spécifiques au Nouveau Roman, dans la mesure où celui-ci se définit par rapport et par opposition au " roman traditionnel », c'est-à-dire aux codes romanesques hérités de Balzac. Comme le souligne Winfried Wehle, le problème du réalisme est « l'enjeu de fond indissolublement lié au roman depuis

35. - W. Krauss, « Révolution des Romans? Bemerkungen zum "Nouveau Roman"»...,op. cit., p. 117.

36. - Ibid., p. 119, 127.

37. - Carola Gerlach, Suche nach Realismus im Nouveau Roman am Beispiel der frühen Texte von Alain Robbe-Grillet, 1984. Thèse de doctorat.

38. - M. Rother, Das Problem des Realismus in den Romanen von Alain RobbeGrillet..., op. cit.

39. - Winfried Wehle, " Proteus im Spiegel. Zum "reflexiven Realismus" des Nouveau Roman ", in : Winfried Wehle (dir.), Französischer Roman der Gegenwart: Erzählstruktur und Wirklichkeit im Nouveau Roman, Berlin, E. Schmidt, 1972.

40. - Brigitte Burmeister, « Nouveau roman und Realismus, Umbruch einer Literaturideologie », Beiträge zur Romanischen Philologie Berlin, 17-2, 1978, p. 219225.

41. - Danielle Risterucci-Roudnicky, France-RDA : anatomie d'un transfert littéraire 1949-1990, Bern, Peter Lang, 1999, p. 353. 
le $\mathrm{XIX}^{\mathrm{e}}$ siècle $^{42} »$, mais c'est aussi un faux débat, dans la mesure où « l'imitation de la réalité dans le roman n'est fondamentalement possible que comme fiction ${ }^{43} »$. C'est pour cela que Wehle se montre critique envers la tendance commune aux critiques marxistes et conservatrices de réduire le Nouveau Roman au rang de reflet de son contexte socioculturel : "Quand on réduit la littérature à la reproduction des rapports sociaux, on prive l'œuvre d'art d'une dimension essentielle, celle de son autonomie esthétique ${ }^{4} \gg$.

Une deuxième préoccupation commune des critiques en France, en RFA et en RDA concerne le rapport du Nouveau Roman à son lecteur. La première expérience du Nouveau Roman est en effet celle de l'illisibilité : face à un texte qui abjure tous les repères traditionnels de l'écriture romanesque, comme le héros, l'intrigue, la psychologie, la chronologie, le lecteur ne peut qu'être perdu. Cette difficulté inhérente aux formes de narration développées par les nouveaux romanciers est critiquée de toute part comme la marque d'une littérature élitiste réservée aux initiés et aux milieux académiques. C'est le cas chez Werner Krauss et Brigitte Burmeister à l'Est, qui se situent dans un contexte de politique culturelle dirigée vers les masses, mais aussi chez Gerd Krause et Winfried Wehle à l'Ouest ${ }^{45}$. L'accusation d'hermétisme est tellement récurrente concernant le Nouveau Roman qu'elle finit par l'incarner, comme en témoigne en France la phrase assassine de Pierre Desproges sur Marguerite Duras. Or, de même que le problème du réalisme posait la question de l'autonomie de l'œuvre d'art, il s'agit là d'un trait fondamental de la modernité, à savoir la participation active du récepteur. En effet, le Nouveau Roman n'est pas un produit fini, clos, mais un entrelacs complexe de significations à démêler et à interpréter. En tant que tel, il rencontre dans l'espace germanophone le développement des études de réception par Hans Robert Jauss dans le cadre de l'École de Constance. C'est dans cette perspective que se situe Klaus Netzer dans son livre Der Leser des Nouveau Roman ${ }^{46}$. Côté Est également, une attention accrue est accordée au lecteur ainsi que le souligne Danielle Risterucci-Roudnicky dans son étude du contexte de la réception du Nouveau Roman en RDA : " Au milieu des années 1960, une évolution est perceptible dans la théorie littéraire, la position de Lukács est

42. - W. Wehle, «Proteus im Spiegel... », op. cit. p. 3.

43. - Ibid., p. 4.

44. - Winfried Wehle, Französischer Roman der Gegenwart: Erzählstruktur und Wirklichkeit im Nouveau Roman, Berlin, Erich Schmidt, 1972, p. 21.

45. - W. Wehle, « Proteus im Spiegel... », op. cit., p. 19.

46. - Klaus Netzer, Der Leser des Nouveau Roman, Frankfurt a.M., Athenäum, 1970. 
abandonnée au profit de celle de Brecht qui accorde une place centrale à l'“acte" de lecture et au lecteur 47 ».

Cette évolution de la théorie littéraire est-allemande la met en porteà-faux face aux attentes de la politique culturelle officielle, toutefois un rapprochement paraît possible à certaines conditions :

Si le réalisme socialiste n'est en aucun cas remis en cause et si les expériences des modernes sont toujours condamnées, il apparaît néanmoins que l'on intègre progressivement l'idée d'un rapport entre la nouveauté de la réalité et la nouveauté de certaines formes littéraires, du moment que l'idéologie se trouve respectée (l'idéal humain socialiste $)^{48}$.

Le problème, c'est que le Nouveau Roman ne remplit pas ces conditions : il tombe largement sous le coup de l'accusation d'« antihumanisme » adressée à toute littérature moderne, considérée par Alfred Kurella comme décadente.

De manière générale, la réception du Nouveau Roman en France, en RFA et en RDA présente toujours le même souci quant à la disparition de l'humain, notamment chez Alain Robbe-Grillet. Uwe Neumann montre comment les critiques est-allemands et ouest-allemands, et jusqu’à Lukács en personne, reprochent au Nouveau Roman une vision déshumanisée du monde et un refus de l'engagement ${ }^{49}$. Le principal intéressé se montre particulièrement remonté lorsqu'il se retrouve sous le feu de critiques au colloque de Leningrad de 1963 et ne manque pas de renvoyer ses détracteurs dos à dos :

Mais je voudrais dire mon étonnement d'avoir retrouvé, dans la bouche de la plupart des orateurs soviétiques, des critiques très vives adressées aux recherches de la littérature moderne, ressemblant exactement aux critiques que l'on nous fait dans la société bourgeoise d'Occident. Ici comme là, on nous reproche la « gratuité » et le « formalisme ». On juge notre art « décadent » et « inhumain ». Les questions que l'on nous pose sont: «Pourquoi écrivez-vous? À quoi servez-vous? Quelle est votre fonction dans la société ?»

Naturellement, ces questions sont absurdes. [...]

Mais ce qui nous paraît le plus scandaleux, c'est de retrouver ainsi dans le camp socialiste et dans le monde bourgeois les mêmes illusions quant au pouvoir politique de l'art, le même culte des formes artistiques périmées, le même vocabulaire critique, et en fin de compte les mêmes valeurs. [...]

47. - D. Risterucci-Roudnicky, France-RDA..., op.cit., p. 364.

48. - Ibid., p. 367.

49. - Cf. U. Neumann, Uwe Johnson und der Nouveau roman..., op. cit., p. 476478. 
«Inhumain » ? N'est-ce pas plutôt que votre conception de l'homme demande à être un peu renouvelée 50 ?

\section{Conclusion}

Le Nouveau Roman s'avère être une référence incontournable des discours sur la modernité, aussi bien en RDA qu'en RFA, il fonctionne comme une véritable pierre de touche de la modernité littéraire. Dans son livre de 1977, Zwei deutsche Literaturen?, Eberhard Mannack fait même le détour par le Nouveau Roman pour répondre à la question de l'unité de la littérature allemande entre Est et Ouest. L'étude du transfert du Nouveau Roman dans l'Allemagne divisée a permis de mettre en lumière le rôle des pays périphériques (l'Autriche et la Suisse pour la RFA, les pays du bloc de l'Est pour la RDA) ainsi que certaines figures importantes de passeurs. Elle a aussi montré toute l'ambivalence de la réception d'un mouvement littéraire dont la position de fer de lance de la modernité a été reconnue très tôt, mais qui n'a jamais pu être tout à fait assimilé aux contextes d'accueil. Sur les questions du réalisme, du rapport au lecteur et de l'humanisme, on constate que les réticences de la critique sont communes à l'Est et à l'Ouest, bien qu'elles fonctionnent dans des cadres culturels et théoriques différents. Or, ces questions ont toutes trait à des caractéristiques essentielles de la modernité littéraire : le refus de la mimésis, l'auto-référentialité, l'ouverture interprétative, l'autonomie de l'œuvre d'art. Si les prises de position diffèrent, elles sont donc pareillement assujetties au discours dominant sur la modernité.

50. - A. Robbe-Grillet, «L'écrivain, par définition, ne sait où il va, et il écrit pour chercher à comprendre pourquoi il écrit », op. cit., p. 63-65. 